\title{
Symmetry Operators and Separation of Variables for Dirac's Equation on Two-Dimensional Spin Manifolds ${ }^{\star}$
}

\author{
Alberto CARIGNANO ${ }^{\dagger^{1}}$, Lorenzo FATIBENE $\dagger^{2}$, Raymond G. McLENAGHAN $\dagger^{3}$ \\ and Giovanni RASTELLI ${ }^{\dagger}$ \\ $\dagger^{1}$ Department of Engineering, University of Cambridge, United Kingdom \\ E-mail: ac737@cam.ac.uk \\ $\dagger^{2}$ Dipartimento di Matematica, Università di Torino, Italy \\ E-mail: lorenzo.fatibene@unito.it \\ $\dagger^{3}$ Department of Applied Mathematics, University of Waterloo, Ontario, Canada \\ E-mail: rgmclena@uwaterloo.ca \\ $\dagger^{4}$ Formerly at Dipartimento di Matematica, Università di Torino, Italy \\ E-mail: giorast.giorast@alice.it
}

Received February 01, 2011, in final form June 02, 2011; Published online June 15, 2011

doi:10.3842/SIGMA.2011.057

\begin{abstract}
A signature independent formalism is created and utilized to determine the general second-order symmetry operators for Dirac's equation on two-dimensional Lorentzian spin manifolds. The formalism is used to characterize the orthonormal frames and metrics that permit the solution of Dirac's equation by separation of variables in the case where a second-order symmetry operator underlies the separation. Separation of variables in complex variables on two-dimensional Minkowski space is also considered.
\end{abstract}

Key words: Dirac equation; symmetry operators; separation of variables

2010 Mathematics Subject Classification: 70S10; 81Q80

This paper is dedicated to Professor Willard Miller, Jr. on the occasion of his retirement from the School of Mathematics at the University of Minnesota.

\section{Introduction}

This paper is a contribution to the study of the separability theory for Dirac's equation to which Professor Miller has made important contributions [17, 18, 24]. Exact solutions to Dirac's relativistic wave equation by means of the method of separation of variables have been studied since the equation was postulated in 1928. Indeed, the solution for the hydrogen atom may be obtained by this method. While there is a well developed theory of separation of variables for the Hamilton-Jacobi equation, and the Schrödinger equation based on the existence of valence two characteristic Killing tensors which define respectively quadratic first integrals and second-order symmetry operators for these equations (see [23, 16, 1, 12]) an analogous theory for the Dirac equation is still in its early stages. The complications arise from the fact that one is dealing with a system of first-order partial differential equations whose derivation from the invariant Dirac equation depends not only on the choice of coordinate system but also on the choice of an orthonormal moving frame and representation for the Dirac matrices with respect to which the components of the unknown spinor are defined. Further complications arise if the background

\footnotetext{
*This paper is a contribution to the Special Issue "Symmetry, Separation, Super-integrability and Special Functions $\left(\mathrm{S}^{4}\right)$ ". The full collection is available at http://www.emis.de/journals/SIGMA/S4.html
} 
space-time is assumed to be curved. Much of the progress in the theory has been stimulated by developments in Einstein's general theory of relativity where one studies first quantized relativistic electrons on curved background space-times of physical interest such as the Schwarzschild and Kerr black hole space-times. This work required the preliminary development of a theory of spinors on general pseudo-Riemmanian manifolds (see $[9,5,10,11]$ ). The solution of the Dirac equation in the Reissner-Nordstrom solution was apparently first obtained by Brill and Wheeler in 1957 [2] who separated the equations for the spinor components in standard orthogonal Schwarzschild coordinates with respect to a moving frame adapted to the coordinate curves. A comparable separable solution in the Kerr solution was found by Chandrasekhar in 1976 [6] by use of an ingenious separation ansatz involving Boyer-Lindquist coordinates and the Kinnersley tetrad. The separability property was characterized invariantly by Carter and McLenaghan [4] in terms of a first-order differential operator constructed from the valence two Yano-Killing tensor that exists in the Kerr solution, that commutes with the Dirac operator and that admits the separable solutions as eigenfunctions with the separation constant as eigenvalue. Study of this example led Miller [24] to propose the theory of a factorizable system for first-order systems of Dirac type in the context of which the separability property may be characterized by the existence of a certain system of commuting first-order symmetry operators. While this theory includes the Dirac equation on the Kerr solution and its generalizations [15] it is apparently not complete since as is shown by Fels and Kamran [15] there exist systems of the Dirac type whose separability is characterized by second-order symmetry operators. The work begun by these authors has been continued by Smith [25], Fatibene, McLenaghan and Smith [13], McLenaghan and Rastelli [20], and Fatibene, McLenaghan, Rastelli and Smith [14] who studied the problem in the simplest possible setting namely on two-dimensional Riemmanian spin manifolds. The motivation for working in the lowest permitted dimension is that it is possible to examine in detail the different possible scenarios that arise from the separation ansatz and the imposition of the separation paradigm that the separation be characterized by a symmetry operator admitting the separable solutions as eigenfunctions. The insight obtained from this approach may help suggest an approach to take for the construction of a general separability theory for Dirac type equations. Indeed in [20] systems of two first-order linear partial equations of Dirac type which admit multiplicative separation of variables in some arbitrary coordinate system and whose separation constants are associated with commuting differential operators are exhaustively characterized. The requirement that the original system arises from the Dirac equation on some two-dimensional Riemannian spin manifold allows the local characterization of the orthonormal frames and metrics admitting separation of variables for the equation and the determination of the symmetry operators associated to the separation. The paper [14] takes this research in a different but closely related direction. Following earlier work of McLenaghan and Spindel [22] and Kamran and McLenaghan [19] where the first-order symmetry operators of the Dirac equation where computed on four-dimensional Lorentzian spin manifolds and McLenaghan, Smith and Walker [21] where the second-order operators were determined in terms of a two-component spinor formalism, the most general second-order linear differential operator which commutes with the Dirac operator on a general two-dimensional Riemannian spin manifold is obtained. Further it is shown that the operator is characterized in terms of Killing vectors and valence two Killing tensors defined on the background manifold. The derivation is manifestly covariant: the calculations are done in a general orthonormal frame without the choice of a particular set of Dirac matrices.

The purpose of the present paper is to extend the results of [20] and [14] described above to the case of two-dimensional Lorentzian spin manifolds. One of the main achievements of the paper is the creation of a formalism that permits the simultaneous treatment of both the Riemannian and Lorentzian cases. Further, we extend the results of [8], where Hamilton-Jacobi separability separability is studied in complex variables on two-dimensional Minkowski space, to the Dirac equation. 
The paper is organized as follows. In Section 2 we summarize the basic properties of twodimensional spin manifolds required for the subsequent calculations. Section 3 is devoted to the derivation of the form of the general second-order linear differential operator which commutes with the Dirac operator. We show that this operator is characterized by a valence two Killing tensor field, two Killing vector fields and two scalar fields defined on the background spin-manifold. In Section 4 we develop a formalism based on [20] which enables us to study simultaneously separation of variables for the Dirac equation on both Riemannian and Lorentzian spin manifolds. All possible cases where separation occurs are determined. In Section 5 we establish a link between the second-order symmetry operators obtained formally in Section 3 and the second-order symmetry operator underlying the separation of variables scheme considered in Section 4. In Section 6 separation of Dirac's equation in complex variables is considered on twodimensional Minkowski space. Section 7 contains the appendix. The notation and conventions of this paper are consistent with [14].

\section{Framework}

Let $M$ be a connected, paracompact, two-dimensional spin manifold. Let us consider both the Euclidean $\eta=(2,0)$ and the Lorentzian $\eta=(1,1)$ signatures. With an abuse of notation, let $\eta$ also denote the canonical form induced by the signature and the determinant of such quadratic form, namely one has $\eta= \pm 1$. We will keep this sign as an undetermined parameter in this paper, since we want to consider both cases at once.

We know that a representation of the Clifford algebra is induced by a set of Dirac matrices $\gamma_{a}$ such that they satisfy the Dirac condition

$$
\gamma_{a} \gamma_{b}+\gamma_{b} \gamma_{a}=2 \eta_{a b} I
$$

The generators of the even Clifford algebra are $I, \gamma_{a}$ and $\gamma:=\gamma_{1} \gamma_{2}$. Therefore the most general element of the $\operatorname{Spin}(\eta)$ group is

$$
S=a I+b \gamma
$$

with $a^{2}+\eta b^{2}=1$.

From the theory, we know that it is possible to define a covering map between $\operatorname{Spin}(\eta)$ and $\mathrm{SO}(\eta)$. Let $l_{b}^{a}$ be a generic element of $\mathrm{SO}(\eta)$. Then

$$
\eta_{a b}=l_{a}^{c} \eta_{c d} l_{b}^{d}
$$

and the covering map in matrix form is

$$
l(S)=\left(\begin{array}{cc}
a^{2}-\eta b^{2} & 2 \eta a b \\
-2 \eta a b & a^{2}-\eta b^{2}
\end{array}\right) .
$$

Let $P \rightarrow M$ be a suitable $\operatorname{Spin}(\eta)$ principal bundle, such that it allows global maps $\Lambda: P \rightarrow$ $L(M)$ of the spin bundle into the general frame bundle of $M$. The local expression of such maps is given by spin frames $e_{a}^{\mu}$.

A spin frame induces a metric of signature $\eta$ and the corresponding spin connection:

$$
g_{\mu \nu}=e_{\mu}^{a} \eta_{a b} e_{\nu}^{b}, \quad \Gamma_{\mu}^{a b}=e_{\alpha}^{a}\left(\Gamma_{\beta \mu}^{\alpha} e^{b \beta}+\partial_{\mu} e_{c}^{\alpha} \eta^{c b}\right),
$$

where $\Gamma_{\beta \mu}^{\alpha}$ denotes the Levi-Civita connection of the induced metric $g_{\mu \nu}$, which in turn induces the covariant derivative as $\nabla_{\mu}=e_{\mu}^{a} \nabla_{a}$.

It follows from our setting that the inner product $\eta_{a b}$ will raise and lower Latin indices, while the Greek ones are raised and lowered by the induced metric $g_{\mu \nu}$. 
Finally, the covariant derivative of spinors is defined as

$$
\nabla_{\mu} \psi:=\partial_{\mu} \psi+\Gamma_{\mu} \psi
$$

where

$$
\Gamma_{\mu}=\frac{1}{8} \Gamma_{\mu}^{a b}\left[\gamma_{a}, \gamma_{b}\right]=\frac{1}{4} \Gamma_{\mu}^{a b} \epsilon_{a b} \gamma
$$

The covariant derivative (1) is invariant under spin transformations in view of the following property

$$
S \partial_{\nu} S^{-1}=\frac{1}{4} l_{a}^{b} \partial_{\nu} \bar{l}_{c}^{a} \epsilon_{b}^{c} \gamma
$$

A spin transformation is an automorphism of the spin bundle $P$. If we define the fiber coordinates of $P$ as $(x, S)$, the local expressions of the spin transformation are $x^{\prime \mu}=x^{\prime \mu}(x)$, $S^{\prime}=\phi(x) S$, where $\phi: U \rightarrow \operatorname{Spin}(\eta)$.

As we know, it acts as a left group on spinors and spin frames $\left(l_{a}^{b} \equiv l_{a}^{b}(\varphi)\right)$

$$
\psi^{\prime}=\phi \psi, \quad e_{a}^{\prime \mu}=J_{\nu}^{\mu} e_{b}^{\nu} \bar{l}_{a}^{b}
$$

where $l: \operatorname{Spin}(\eta) \rightarrow \mathrm{SO}(\eta)$ and $J_{\nu}^{\mu}$ is the Jacobian of the coordinate transformation. Therefore the spin connection transforms as

$$
\Gamma_{\mu}^{\prime a b}=\bar{J}_{\mu}^{\nu} l_{c}^{a}(\psi)\left(\Gamma_{\nu}^{c d} l_{d}^{b}(\psi)+\partial_{\nu} \bar{l}_{d}^{c}(\psi) \eta^{d b}\right) .
$$

Looking back to the covariant derivative, one can prove that the commutator can be related to the curvature

$$
\left[\nabla_{\mu}, \nabla_{\nu}\right] \psi=\frac{1}{4} \gamma \psi \epsilon_{a b} R_{\mu \nu}^{a b}
$$

The advantage of working in dimension two is that the Riemann tensor $R_{\mu \nu}^{\alpha \beta}$ has only one independent component that can be written as a function of the Ricci scalar $R$. Hence the identity (2) may be rewritten as

$$
\left[\nabla_{c}, \nabla_{d}\right] \psi=\frac{1}{4} \gamma \psi \epsilon_{c d} R
$$

Similarly the following property holds

$$
\left[\nabla_{a}, \nabla_{b}\right] \nabla_{c} \psi=\frac{R}{4} \gamma \epsilon_{a b} \nabla_{c} \psi-\frac{R}{2} \epsilon_{c}^{d \cdot} \epsilon_{a b} \nabla_{d} \psi
$$

\section{First- and second-order symmetry operators}

In our framework, the Dirac equation has the form

$$
\mathbb{D} \psi=i \gamma^{a} \nabla_{a} \psi-m \psi=0 .
$$

An operator is a symmetry operator for the Dirac equation if

$$
[\mathbb{K}, \mathbb{D}]=0 .
$$

The most general operator of the second-order has the form

$$
\mathbb{K}=\mathbb{E}^{a b} \nabla_{a b}+\mathbb{F}^{a} \nabla_{a}+\mathbb{G} I,
$$

where $\mathbb{E}^{a b}, \mathbb{F}^{a}, \mathbb{G}$ are algebraic matrix coefficients to be determined. 
We aim to determine coefficients so that condition (6) holds true. Considering the Ricci's identities (2), (3) and (4), we expand the symmetry equation (6) for the coefficients and we obtain

$$
\begin{aligned}
& \mathbb{E}^{(a b} \gamma^{c)}-\gamma^{(c} \mathbb{E}^{a b)}=0, \quad \mathbb{F}^{(a} \gamma^{b)}-\gamma^{(b} \mathbb{F}^{a)}=\gamma^{c} \nabla_{c} \mathbb{E}^{a b}, \\
& \mathbb{G} \gamma^{d}-\gamma^{d} \mathbb{G}=\gamma^{c} \nabla_{c} \mathbb{F}^{d}-\left(\mathbb{E}^{a d} \gamma^{c}+\gamma^{c} \mathbb{E}^{a d}\right) \gamma \frac{R}{4} \epsilon_{a c}++\frac{R}{3}\left(\frac{1}{2} \mathbb{E}^{b a} \gamma^{c}+\gamma^{c} \mathbb{E}^{b a}\right) \epsilon_{a}^{d \cdot} \epsilon_{b c}, \\
& \gamma^{c} \nabla_{c} \mathbb{G}=\frac{R}{8}\left(\mathbb{F}^{a} \gamma^{c}+\gamma^{c} \mathbb{F}^{a}\right) \gamma \epsilon_{a c}+\left(\frac{1}{2} \gamma^{c} \mathbb{E}^{a b}+\mathbb{E}^{a b} \gamma^{c}\right) \frac{1}{6} \gamma \epsilon_{b c} \nabla_{b} R .
\end{aligned}
$$

To write the system (7) we make use of a characterization of second- and third-order covariant derivatives which is shown in the Appendix.

Let us begin with the first equation in (7). We know that the coefficients of $\mathbb{E}^{a b}$ are zero-order matrix operators (i.e. not differential) which can be expanded in the basis of $C(\eta)$

$$
\mathbb{E}^{a b}=e^{a b} I+e_{c}^{a b} \gamma^{c}+\hat{e}^{a b} \gamma
$$

where the coefficients are point functions in $M$. Here we are using the fact that $I, \gamma^{c}$ and $\gamma$ form a basis, since they are linearly independent. Hence, the first equation can be rewritten as

$$
-2 \hat{e}^{(a b} \epsilon_{d}^{c) \cdot} \gamma^{d}-2 \eta e_{d}^{(a b} \epsilon^{d c)} \gamma=0,
$$

which can be solved to obtain

$$
\hat{e}^{a b}=0, \quad e_{d}^{a b} \gamma^{d}=2 \alpha^{(a} \gamma^{b)},
$$

where $\alpha^{a}$ are the coefficients along the frame $e_{a}:=e_{a}^{\mu} \partial_{\mu}$ of an arbitrary vector field $\alpha$.

Applying these conditions, the operator $\mathbb{E}$ is of the form

$$
\mathbb{E}^{a b}=e^{a b} I+2 \alpha^{(a} \gamma^{b)} .
$$

Now we consider the second condition of (7). As we did for $\mathbb{E}$, we expand $\mathbb{F}$ in the basis

$$
\mathbb{F}^{a}=f^{a} I+f_{b}^{a} \gamma^{b}+\hat{f}^{a} \gamma
$$

and substitute it back in the condition (7). We obtain

$$
\nabla_{c} \alpha^{(a} \eta^{b) c}=0, \quad f_{c}^{(a} \epsilon^{b) c}=\nabla_{c} \alpha^{(a} \epsilon^{b) c}, \quad-2 \hat{f}^{(a} \epsilon_{c}^{b) \cdot}=\nabla_{c} e^{a b} .
$$

The first equation says that $\alpha^{a}$ is a Killing vector of $g$ or possibly the zero vector. Through an explicit calculation, we prove that the most general tensor satisfying the second condition is $A \delta_{c}^{a}$. Hence

$$
f_{c}^{a}=\nabla_{c} \alpha^{a}+A \delta_{c}^{a} .
$$

Finally, we substitute into the third equation of (8) obtaining

$$
-2 \hat{f}^{(a} \epsilon_{c}^{b) \cdot} \epsilon^{c} \cdot d=\nabla_{c} e^{a b} \epsilon_{\cdot d}^{c} .
$$

Taking the trace we get

$$
\hat{f}^{b}=\frac{1}{3} \nabla_{c} e^{a b} \epsilon_{\cdot a}^{c}=\frac{1}{3} \nabla^{c} e^{a b} \epsilon_{c a},
$$

which shows that $\hat{f}^{a}$ is uniquely determined by $e^{a c}$. However (9) contains six equations, of which only one has been used. The other five equations are exploited by back substituting (10) into (9) to obtain an equation for $e^{a c}$ alone, namely

$$
\nabla^{c} e^{e a} \epsilon_{c e} \delta_{d}^{b}+\nabla^{c} e^{e b} \epsilon_{c e} \delta_{d}^{a}=\nabla_{c} e^{a b} \epsilon_{\cdot d}^{c} .
$$


This is an integrability condition for $e^{a b}$ which may be written as

$$
\nabla^{(a} e^{b c)}=0 .
$$

This equation shows that $e^{a b}$ is a Killing tensor of $g$.

We shall now consider the third condition of (7)

$$
\mathbb{G} \gamma^{d}-\gamma^{d} \mathbb{G}=\gamma^{c} \nabla_{c} \mathbb{F}^{d}-\left(\mathbb{E}^{a d} \gamma^{c}+\gamma^{c} \mathbb{E}^{a d}\right) \gamma \frac{R}{4} \epsilon_{a c}+\frac{R}{3}\left(\frac{1}{2} \mathbb{E}^{b a} \gamma^{c}+\gamma^{c} \mathbb{E}^{b a}\right) \epsilon_{a}^{d \cdot} \epsilon_{b c} .
$$

Considering the usual expansion $\mathbb{G}=g I+g_{a} \gamma^{a}+\hat{g} \gamma$, we obtain the following system of equations

$$
\begin{aligned}
& 0=\nabla_{a} A-\frac{R}{2} \alpha^{a} I+\frac{R}{2} \alpha^{a} I, \\
& 2 g_{b} \epsilon_{. .}^{b a}=\left(\nabla_{b} A-\frac{R}{2} \alpha_{b}\right) \epsilon_{. .}^{b a}-\frac{R}{2} \alpha_{b} \epsilon_{. .}^{b a}-\frac{R}{2} \alpha_{b} \epsilon_{. .}^{a b}, \\
& -2 \eta \hat{g} \epsilon_{. .}^{a b} \gamma^{b}=\nabla_{b} f^{a}+\frac{\eta}{3} \epsilon_{d c} \epsilon_{e .}^{b} \nabla^{e} \nabla^{d} e^{a c}+\frac{R}{2} e^{a b}+\frac{R}{2} e^{c d} \epsilon_{d}^{a \cdot} \epsilon_{c .}^{b} .
\end{aligned}
$$

The first equation means that $A \in \mathbb{C}$, while the second implies $g_{b}=-\frac{R}{4} \alpha_{b}$. By splitting the third into its symmetric and antisymmetric parts, it results that

$$
\hat{g}=\frac{1}{4} \epsilon_{b a} \nabla^{b} f^{a}
$$

for the antisymmetric part, while the symmetric part can be expanded to obtain

$$
\nabla^{(a}\left(f^{b)}-\nabla_{c} e^{b) c}\right)=0 .
$$

It follows that there exists a Killing vector or a zero-vector $\zeta$ such that $\zeta^{a}=f^{a}-\nabla_{c} e^{a c}$ from which we obtain

$$
f^{a}=\zeta^{a}+\nabla_{c} e^{a c} .
$$

We summarize all the results obtained so far

$$
\begin{aligned}
& \mathbb{E}^{a b}=e^{a b} I+2 \alpha^{(a} \gamma^{b)}, \\
& \mathbb{F}^{a}=\left(\zeta^{a}+\nabla_{c} e^{a c}\right) I+\left(\gamma^{c} \nabla_{c} \alpha^{a}+A \gamma^{a}\right)+\frac{1}{3} \epsilon_{b c} \nabla^{b} e^{a c} \gamma, \\
& \mathbb{G}=g I-\frac{R}{4} \alpha_{b} \gamma^{b}+\frac{1}{4} \epsilon_{b a} \nabla^{b} \zeta^{a} \gamma,
\end{aligned}
$$

where

$$
A \in \mathbb{C}, \quad \nabla^{(d} e^{a b)}=0, \quad \nabla^{(a} \alpha^{b)}=0, \quad \nabla^{(d} \zeta^{b)}=0 .
$$

It remains to consider the fourth equation in (7)

$$
\gamma^{c} \nabla_{c} \mathbb{G}=\frac{R}{8}\left(\mathbb{F}^{a} \gamma^{c}+\gamma^{c} \mathbb{F}^{a}\right) \gamma \epsilon_{a c}+\left(\frac{1}{2} \gamma^{c} \mathbb{E}^{a b}+\mathbb{E}^{a b} \gamma^{c}\right) \frac{1}{6} \gamma \epsilon_{b c} \nabla_{b} R
$$

which implies the only additional condition:

$$
\nabla^{a} g=-\frac{1}{4} \nabla_{b}\left(R e^{a b}\right)
$$

This equation locally determines $g$ if and only if the right hand side is a closed 1-form. We call (11) the integrability condition.

We now focus on finding condition for first-order operators.

We can easily obtain the conditions by setting to zero $e^{a b}$ and $\alpha^{a}$. In particular (11) is trivially satisfied and $g \in \mathbb{C}$. We thus obtain that the most general first-order symmetry operator may be written as

$$
\mathbb{E}^{a b}=0, \quad \mathbb{F}^{a}=\zeta^{a} I+A \gamma^{a}, \quad \mathbb{G}=g I+\frac{1}{4} \epsilon_{b a} \nabla^{b} \zeta^{a} \gamma,
$$

where $A, g \in \mathbb{C}$ and $\nabla^{(d} \zeta^{b)}=0$. 


\section{Separation of variables}

Let us start with the Dirac condition

$$
\gamma^{a} \gamma^{b}+\gamma^{b} \gamma^{a}=2 \eta^{a b} I
$$

We fix the metric convention to be $\eta^{a b}=\operatorname{diag}(1, \pm 1)$.

A choice of gamma matrices valid for both signatures is

$$
\gamma^{1}=\left(\begin{array}{cc}
1 & 0 \\
0 & -1
\end{array}\right), \quad \gamma^{2}=\left(\begin{array}{cc}
0 & -k \\
k & 0
\end{array}\right)
$$

where $k=i$ for Euclidean and $k=1$ for Lorentzian signature.

Using the gamma matrices and (1), we can rewrite Dirac's equations (5) as

$$
\tilde{A} \partial_{1} \psi+\tilde{B} \partial_{2} \psi+\tilde{C} \psi-\lambda \psi=0
$$

where

$$
\tilde{A}=\left(\begin{array}{cc}
A_{1} & A_{2} \\
-A_{2} & -A_{1}
\end{array}\right), \quad \tilde{B}=\left(\begin{array}{cc}
B_{1} & B_{2} \\
-B_{2} & -B_{1}
\end{array}\right), \quad \tilde{C}=\left(\begin{array}{cc}
C_{1} & -C_{2} \\
C_{2} & -C_{1}
\end{array}\right),
$$

and

$$
\begin{aligned}
& A_{1}=i e_{1}^{1}, \quad A_{2}=-i k e_{2}^{1}, \quad B_{1}=i e_{1}^{2}, \quad B_{2}=-i k e_{2}^{2}, \\
& C_{1}=-\frac{i}{2} k e_{2}^{\mu} \Gamma_{\mu}^{12}, \quad C_{2}=-\frac{i}{2} e_{1}^{\mu} \Gamma_{\mu}^{12} .
\end{aligned}
$$

Let $(x, y)$ be a coordinate system on the two-dimensional manifold and

$$
\psi=\left(\begin{array}{l}
\psi_{1}(x, y) \\
\psi_{2}(x, y)
\end{array}\right)
$$

We now make the important assumption that the spinor $\psi$ is multiplicatively separable

$$
\psi_{i}=a_{i}(x) b_{i}(y)
$$

The Dirac equation then reads:

$$
\begin{aligned}
& A_{1} \dot{a}_{1} b_{1}+A_{2} \dot{a}_{2} b_{2}+B_{1} a_{1} \dot{b}_{1}+B_{2} a_{2} \dot{b}_{2}+C_{1} a_{1} b_{1}-C_{2} a_{2} b_{2}-\lambda a_{1} b_{1}=0, \\
& -A_{2} \dot{a}_{1} b_{1}-A_{1} \dot{a}_{2} b_{2}-B_{2} a_{1} \dot{b}_{1}-B_{1} a_{2} \dot{b}_{2}+C_{2} a_{1} b_{1}-C_{1} a_{2} b_{2}-\lambda a_{2} b_{2}=0 .
\end{aligned}
$$

We now apply the general results of [20] to the Lorentzian case.

Definition 1. The Dirac equation (13) and the operator $\mathbb{D}$ are separated in $(x, y)$ if there exists nonzero functions $R_{i}(x, y)$ such that (13) can be rewritten as

$$
R_{1} a_{r} b_{s}\left(E_{1}^{x}+E_{1}^{y}\right)=0, \quad R_{2} a_{t} b_{u}\left(E_{2}^{x}+E_{2}^{y}\right)=0
$$

for suitable indices $r, s, t, u$ where $E_{i}^{x}\left(x, a_{j}, \dot{a}_{j}\right), E_{i}^{y}\left(y, b_{j}, \dot{b}_{j}\right)$. Moreover, the equations

$$
E_{i}^{x}=\mu_{i}=-E_{i}^{y}
$$

define the separation constants $\mu_{i}$. 
The above definition refers to so-called "naive" separation of variables that is not always the most general. In order to find symmetry operators, we adopt for our analysis some assumptions.

First of all, we assume a given coordinate system $(x, y)$ and impose a separation of (13) according to the previous definition. This can be done in three different ways

Type I: $a_{1} \neq a_{2}$ and $b_{1} \neq b_{2}$.

Type II: $a_{1}=a_{2}$ and $b_{1} \neq b_{2}$ (or viceversa).

Type III: $a_{1}=c a_{2}=a$ and $b_{1}=d b_{2}=b(c, d \in \mathbb{C})$.

Following the procedure laid out in [20], we build eigenvalue-type operators $\mathbb{L} \psi=\mu \psi$ with eigenvalues $\mu\left(\mu_{i}\right)$ making use of the terms $E_{i}^{x}$ and $E_{i}^{y}$ in (15) only. We require that the operators $\mathbb{L}$ are independent of $\lambda$. Furthermore, $\lambda \neq 0$.

Finally, we require the symmetry condition, that is $[\mathbb{L}, \mathbb{D}] \psi=(\mathbb{L} \mathbb{D}-\mathbb{D L}) \psi=0$ for all $\psi$. A operator $\mathbb{L}$ which satisfies the condition, is called a symmetry operator since it maps solutions into solutions.

The symmetry operators are directly generated by the separated equations and having them enables one to immediately write down the same separated equations. In addition, the separation constants are associated with eigenvalues of symmetry operators.

The only relevant case is Type I separation, since it is the only one associated with nontrivial second-order operators. We shall now make use of the naive separation assumption and of definition (14). We would like to determine the indices $r, s, t, u$ and $E_{i}^{x}\left(x, a_{j}, \dot{a}_{j}\right), E_{i}^{y}\left(y, b_{j}, \dot{b}_{j}\right)$. Thus, we focus on what we can factorize from (13).

By inspection, we notice that the only allowed factorizations are:

- Factorize $a_{1} b_{2}$ in the first equation and $a_{2} b_{1}$ in the second equation.

- Factorize $a_{2} b_{1}$ in the first equation and $a_{1} b_{2}$ in the second equation.

We consider the first factorization, which implies $A_{1}=B_{2}=0$. We divide into two parts both the equations: one part which is a function of $x$ and the other a function of $y$, in order to have $E_{1}^{x}, E_{1}^{y}$ and $E_{2}^{x}, E_{2}^{y}$.

Hence

$$
E_{1}^{x}=A_{2} R_{1} \frac{\dot{a_{2}}}{a_{1}}-C_{2} R_{1} \frac{a_{2}}{a_{1}}=\mu_{1}, \quad E_{1}^{y}=B_{1} R_{1} \frac{\dot{b_{1}}}{b_{2}}+C_{1} R_{1} \frac{b_{1}}{b_{2}}-\lambda R_{1} \frac{b_{1}}{b_{2}}=-\mu_{1},
$$

and

$$
E_{2}^{x}=A_{2} R_{2} \frac{\dot{a_{1}}}{a_{2}}-C_{2} R_{2} \frac{a_{1}}{a_{2}}=\mu_{2}, \quad E_{2}^{y}=B_{1} R_{2} \frac{\dot{b_{2}}}{b_{1}}+C_{1} R_{2} \frac{b_{2}}{b_{1}}+\lambda R_{2} \frac{b_{2}}{b_{1}}=-\mu_{2}
$$

where $\mu_{1}$ and $\mu_{2}$ are the separation constants.

The second-order operator $\mathbb{L}$ is therefore defined by the following equations

$$
A_{2} R_{1} \dot{a_{2}}-C_{2} R_{1} a_{2}=\mu_{1} a_{1}, \quad A_{2} R_{1} \dot{a_{1}}-C_{2} R_{1} a_{1}=\mu_{2} a_{2} .
$$

If we set $\mu_{1} \mu_{2}=\mu$, the operator is given by

$$
\begin{aligned}
\mathbb{L} \psi:= & \left(\begin{array}{cc}
A_{2}^{2}(x, y) R_{1}^{2}(y) & 0 \\
0 & A_{2}^{2}(x, y) R_{1}^{2}(y)
\end{array}\right) \partial_{x}^{2} \psi \\
& +\left(\begin{array}{cc}
A_{2} R_{1}^{2} \partial_{x} A_{2}-C_{2} A_{2} R_{1}^{2}-C_{2} R_{1}^{2} A_{2} & A_{2} R_{1}^{2} \partial_{x} A_{2}-C_{2} A_{2} R_{1}^{2}-C_{2} R_{1}^{2} A_{2}
\end{array}\right) \partial_{x} \psi \\
0 &
\end{aligned}
$$




$$
+\left(\begin{array}{cc}
C_{2}^{2}(x, y) R_{1}^{2}(y) & 0 \\
-A_{2}(x, y) R_{1}^{2}(y) \partial_{x} C_{2}(x, y) & C_{2}^{2}(x, y) R_{1}^{2}(y) \\
0 & -A_{2}(x, y) R_{1}^{2}(y) \partial_{x} C_{2}(x, y)
\end{array}\right) \psi=\mu \psi
$$

We notice that the functions $A_{2} R_{1}$ and $C_{2} R_{1}$ are functions only of $x$.

Finally, we look for the conditions that have to be applied in order to have the operator commuting with the Dirac operator $D$ : it results already that $[\mathbb{L}, \mathbb{D}]=0$, so no other conditions are needed. It follows from a detailed analysis of this case (D5 separation scheme in [20]) that we obtain a Liouville metric with one ignorable coordinate. This case will be discussed in detail in the next section. Another case is also possible which corresponds to the D7 separation scheme in [20]. However, it may be shown that it is equivalent to previous one modulo the sign of the Lorentz metric.

Separability for equations of Type II and of Type III gives rise to first-order operators.

\section{$5 \quad$ Liouville metric}

In Section 2 we concluded our analysis of symmetry operators with the condition (11) on the second-order operator:

$$
\nabla^{a} g=-\frac{1}{4} \nabla_{b}\left(R e^{a b}\right)
$$

Its analysis requires knowledge regarding which spin manifolds $M$ admit nontrivial valence two Killing tensors.

To further proceed with our analysis, we recall the following important result.

A two-dimensional Riemannian or Lorentzian space admits a non-trivial valence two Killing tensor if and only if it is a Liouville surface in which case there exists a system of coordinates $(u, v)$ with respect to which the metric $g$ and Killing tensor $K$ have the following forms

$$
\begin{aligned}
& g=(A(u)+B(v))\left(d u^{2}+\eta d v^{2}\right), \\
& K=\frac{B(v)}{A(u)+B(v)} \partial_{u} \otimes \partial_{u}-\eta \frac{A(u)}{A(u)+B(v)} \partial_{v} \otimes \partial_{v}=K^{a b} e_{a} \otimes e_{b},
\end{aligned}
$$

where $A$ and $B$ are arbitrary smooth functions. Furthermore, the frame component of $K$ are given by

$$
\left[K^{a b}\right]=\operatorname{diag}(B(v),-\eta A(u)) .
$$

The spin frame corresponding to D5 separation discussed in the previous section is given by

$$
\left(e_{a}^{\mu}\right)=\left(\begin{array}{cc}
0 & \frac{1}{\sqrt{A(u)+B(v)}} \\
-\frac{1}{\sqrt{A(u)+B(v)}} & 0
\end{array}\right),
$$

where $A=0$ and $B(v)=1 / R_{1}(v)^{2}$.

It follows from the previous section that the only Type I separation, other than the equivalent one discussed at the end of the previous section, is associated with the nonsingular Dirac operator and associated symmetry operator of the form

$$
\mathbb{D}:=R_{1}(y)\left[\left(\begin{array}{cc}
0 & k \\
-k & 0
\end{array}\right) \partial_{x}+i\left(\begin{array}{cc}
1 & 0 \\
0 & -1
\end{array}\right) \partial_{y}\right]+\frac{i}{2} R_{1}^{\prime}(y)\left(\begin{array}{cc}
1 & 0 \\
0 & -1
\end{array}\right), \quad \mathbb{K}=\left(\begin{array}{cc}
\partial_{x}^{2} & 0 \\
0 & \partial_{x}^{2}
\end{array}\right) .
$$


The operator $\mathbb{K}$ written above agrees with the second-order operator of Section 3 computed for the Liouville metric under the assumption $\alpha=\zeta=0$. We observe that it is in fact the square of the first-order operator

$$
\mathbb{L}=\left(\begin{array}{ll}
1 & 0 \\
0 & 1
\end{array}\right) \partial_{x}
$$

corresponding to the Killing vector associated to the ignorable coordinate $x$ of the Liouville metric where, as we said before, $k=i$ for Euclidean and $k=1$ for Lorentzian signature.

The corresponding coordinates separate the geodesic Hamilton-Jacobi equation. If the manifold is the Euclidean plane, the coordinates, up to a rescaling, coincide with polar or Cartesian coordinates. In the Minkowski plane the coordinates correspond to pseudo-Euclidean or pseudopolar coordinates.

\section{Separation in complex variables}

On real pseudo-Riemannian manifolds the Hamilton-Jacobi equation can be separated not only in standard separable coordinates but also in complex variables [8]. As in classical separation of variables theory, complex separable variables are determined by eigenvalues and eigenvectors of second-order Killing tensors. If the manifold is pseudo-Riemannian, pairs of complex-conjugate eigenvectors and eigenvalues of second-order real Killing tensors can exist in some part of the manifold, together with real ones. Where complex eigenvectors appear, it is impossible to determine real orthogonal separable coordinates, and the introduction of complex variables is necessary. The complex variables behave in all respects as complex coordinates, but they are not independent because of the conjugation relation. In the following their lack of independence will be irrelevant. Let us consider the 2-dimensional Minkowski manifold with pseudo-Cartesian coordinates $(x, y)$. The geodesic Hamiltonian is given by

$$
H=\frac{1}{2}\left(p_{x}^{2}-p_{y}^{2}\right) .
$$

The space of valence two Killing tensors is 6-dimensional and there are ten different types of separable webs real in some part of the space [7]. Another separable web, everywhere complex, is defined by [8]

$$
z=x+i y, \quad \bar{z}=x-i y
$$

and is determined by the eigenvectors of the Killing tensor whose non-null components in $(x, y)$ are

$$
K^{12}=K^{21}=1,
$$

and the associated polynomial first integral is

$$
L=p_{x} p_{y}
$$

By defining the canonical momenta as

$$
P=\frac{1}{2}\left(p_{x}-i p_{y}\right), \quad \bar{P}=\frac{1}{2}\left(p_{x}+i p_{y}\right),
$$

we have

$$
H=P^{2}+\bar{P}^{2}, \quad L=i\left(P^{2}-\bar{P}^{2}\right),
$$


and a real complete separated integral of the Hamilton-Jacobi equation can be determined [8]. Because $(z, \bar{z})$ are both ignorable variables, they should also separate the Dirac equation (indeed, in Minkowski space they are the only complex separable web with at least one ignorable variable). With respect to $(x, y)$ the Dirac operator can be written as

$$
\mathbb{D}=i\left[\left(\begin{array}{ll}
e_{1}^{1} & -e_{2}^{1} \\
e_{2}^{1} & -e_{1}^{1}
\end{array}\right) \partial_{x}+\left(\begin{array}{ll}
e_{1}^{2} & -e_{2}^{2} \\
e_{2}^{2} & -e_{1}^{2}
\end{array}\right) \partial_{y}\right],
$$

where $\left(e_{a}^{\mu}\right)$ are the components of the spin frame. Because the $\left(e_{a}^{\mu}\right)$ can be assumed to be constant, and since

$$
\partial_{z}=\frac{1}{2}\left(\partial_{x}-i \partial_{y}\right), \quad \partial_{\bar{z}}=\frac{1}{2}\left(\partial_{x}+i \partial_{y}\right),
$$

we have

$$
\mathbb{D}=i\left[\left(\begin{array}{ll}
e_{1}^{1}+i e_{1}^{2} & -e_{2}^{1}-i e_{2}^{2} \\
e_{2}^{1}+i e_{2}^{2} & -e_{1}^{1}-i e_{1}^{2}
\end{array}\right) \partial_{z}+\left(\begin{array}{cc}
e_{1}^{1}-i e_{1}^{2} & -e_{2}^{1}+i e_{2}^{2} \\
e_{2}^{1}-i e_{2}^{2} & -e_{1}^{1}+i e_{1}^{2}
\end{array}\right) \partial_{\bar{z}}\right] .
$$

In order to write $\mathbb{D}$ in the form

$$
\mathbb{D}=\left[\left(\begin{array}{cc}
0 & -1 \\
1 & 0
\end{array}\right) \partial_{z}+i\left(\begin{array}{cc}
1 & 0 \\
0 & -1
\end{array}\right) \partial_{\bar{z}}\right]
$$

corresponding to Type I separation in the Minkowski space, the components of $\left(e_{a}^{\mu}\right)$ in $(x, y)$ must be

$$
e_{1}^{1}=\frac{i}{2}, \quad e_{1}^{2}=-\frac{1}{2}, \quad e_{2}^{1}=\frac{1}{2}, \quad e_{2}^{2}=-\frac{i}{2} .
$$

Therefore, by using $\left(e_{a}^{\mu}\right)$ and $\left(e_{\mu}^{a}\right)=\left(e_{a}^{\mu}\right)^{-1}$ for raising and lowering indices,

$$
\left(e_{a}\right)=\frac{1}{2}\left(\partial_{x}+i \partial_{y}, \partial_{x}-i \partial_{y}\right)
$$

It is remarkable that the spin frame base allowing the separation of variables in $(z, \bar{z})$ is essentially coincident with $\left(\partial_{\bar{z}}, \partial_{z}\right)$.

Both $z$ and $\bar{z}$ are ignorable variables, therefore, both

$$
\left(\begin{array}{ll}
1 & 0 \\
0 & 1
\end{array}\right) \partial_{z}^{2} \quad \text { and } \quad\left(\begin{array}{ll}
1 & 0 \\
0 & 1
\end{array}\right) \partial_{\bar{z}}^{2}
$$

can be used as differential operators associated with separation of variables. The integration of the $\psi_{i}=a_{i} b_{i}$ is in all respects the same as for the separation in real coordinates.

\section{Appendix}

System (7) is obtained by using equations that characterize second- and third-order covariant derivatives. Such equations make use of Ricci's identities (3) and (4).

For second-order covariant derivatives the following equation hold

$$
\nabla_{a} \nabla_{c} \psi=\frac{1}{2}\left[\nabla_{a}, \nabla_{c}\right] \psi+\nabla_{a c} \psi=\nabla_{a c} \psi+\frac{R}{8} \epsilon_{a c} \gamma \psi .
$$

This last equation can be rewritten as

$$
\nabla_{c} \nabla_{a} \psi=\nabla_{a} \nabla_{c} \psi+\frac{R}{4} \epsilon_{c a} \gamma \psi
$$


Similar equations for third-order covariant derivative require more calculations and we will show only the main passages

$$
\begin{aligned}
\nabla_{a b} \nabla_{c} \psi & =\frac{1}{6}\left(3 \nabla_{a} \nabla_{b} \nabla_{c}+3 \nabla_{b} \nabla_{a} \nabla c\right) \psi \\
& =\frac{1}{6}\left(\nabla_{a} \nabla_{b} \nabla_{c}+\nabla_{b} \nabla_{a} \nabla c+\nabla_{a} \nabla_{b} \nabla_{c}+2 \nabla_{b} \nabla_{a} \nabla c\right) \psi
\end{aligned}
$$

Now by using (16) and expanding:

$$
\begin{aligned}
\nabla_{a b} \nabla_{c} \psi= & \frac{1}{6}\left(\nabla_{a} \nabla_{b} \nabla_{c}+\nabla_{b} \nabla_{a} \nabla_{c}+\nabla_{a} \nabla_{c} \nabla_{b}+\nabla_{b} \nabla_{c} \nabla_{a}+\nabla_{a} \nabla_{c} \nabla_{b}+\nabla_{b} \nabla_{c} \nabla_{a}\right) \psi \\
& +\frac{1}{12} \nabla_{a} R \epsilon_{b c} \gamma \psi+\frac{1}{12} \nabla_{b} R \epsilon_{a c} \gamma \psi+\frac{1}{12} R \epsilon_{b c} \gamma \nabla_{a} \psi+\frac{R}{12} \epsilon_{a c} \gamma \nabla_{b} \psi
\end{aligned}
$$

Now the first part of the right hand side is just $\nabla_{a b c}$. The second part can be rewritten to obtain

$$
\begin{aligned}
\nabla_{a b} \nabla_{c} \psi= & \nabla_{a b c} \psi+\frac{1}{12}\left(\nabla_{a} R \epsilon_{b c}+\nabla_{b} R \epsilon_{a c}\right) \gamma \psi+\frac{R}{8} \epsilon_{a c} \gamma \nabla_{b} \psi \\
& +\frac{R}{8} \epsilon_{b c} \gamma \nabla_{a}+\frac{R}{12}\left(-\eta_{a c} \nabla_{b}-\eta_{b c} \nabla_{a}+2 \eta_{a b} \nabla_{c}\right) .
\end{aligned}
$$

Equations (17) and (16) together with Ricci's identities are all the tools needed to expand equation (6) in system (7).

\section{Acknowledgements}

The authors wish to thank their reciprocal institutions, the Dipartimento di Matematica, Università di Torino and the Department of Applied Mathematics, University of Waterloo for hospitality during which parts of this paper were written. The research was supported in part by a Discovery Grant from the Natural Sciences and Engineering Research Council of Canada.

\section{References}

[1] Benenti S., Intrinsic characterization of the variable separation in the Hamilton-Jacobi equation, J. Math. Phys. 38 (1997), 6578-6602.

[2] Brill D.R., Wheeler J.A., Interaction of neutrinos and gravitational fields, Rev. Mod. Phys. 29 (1957), 465-479.

[3] Bruce A.T., McLenaghan R.G., Smirnov R.G., A geometrical approach to the problem of integrability of Hamiltonian systems by separation of variables, J. Geom. Phys. 39 (2001), 301-322.

[4] Carter B., McLenaghan R.G., Generalized total angular momentum for the Dirac operator in curved spacetime, Phys. Rev. D 19 (1979), 1093-1097.

[5] Cavaglià M., Fatibene L., Francaviglia M., Two-dimensional dilaton gravity coupled to massless spinors, Classical Quantum Gravity 15 (1998), 3627-3643, hep-th/9801155.

[6] Chandrasekhar S., The mathematical theory of black holes, International Series of Monographs on Physics, Vol. 69, Oxford Science Publications, The Clarendon Press, Oxford University Press, New York, 1983.

[7] Chanu C., Degiovanni L., McLenaghan R.G., Geometrical clasification of Killing tensors on bidimensional flat manifolds, J. Math. Phys. 47 (2006), 073506, 20 pages, math.DG/0512324.

[8] Degiovanni L., Rastelli G., Complex variables for separation of the Hamilton-Jacobi equation on real pseudoRiemannian manifolds, J. Math. Phys. 48 (2007), 073519, 23 pages, nlin.SI/0610012.

[9] Fatibene L., Francaviglia M., Natural and gauge natural formalism for classical field theories. A geometric perspective including spinors and gauge theories, Kluwer Academic Publishers, Dordrecht, 2003.

[10] Fatibene L., Francaviglia M., Deformations of spin structures and gravity, Acta Phys. Polon. B 29 (1998), 915-928.

[11] Fatibene L., Ferraris M., Francaviglia M., Godina M., Gauge formalism for general relativity and fermionic matter, Gen. Relativity Gravitation 30 (1998), 1371-1389, gr-qc/9609042.

[12] Fatibene L., Ferraris M., Francaviglia M., McLenaghan R.G., Generalized symmetries in mechanics and field theories, J. Math. Phys. 43 (2002), 3147-3161. 
[13] Fatibene L., McLenaghan R.G., Smith S., Separation of variables for the Dirac equation on low dimensional spaces, in Advances in General Relativity and Cosmology, Pitagora, Bologna, 2003, 109-127.

[14] Fatibene L., McLenaghan R.G., Rastelli G., Smith S.N., Symmetry operators for Dirac's equation on twodimensional spin manifolds, J. Math. Phys. 50 (2009), 053516, 12 pages, arXiv:0812.2269.

[15] Fels M., Kamran N., Nonfactorizable separable systems and higher-order symmetries of the Dirac operator, Proc. Roy. Soc. London Ser. A 428 (1990), no. 1874, 229-249.

[16] Kalnins E.G., Separation of variables for Riemannian spaces of constant curvature, Pitman Monographs and Surveys in Pure and Applied Mathematics, Vol. 28, Longman Scientific \& Technical, Harlow; John Wiley \& Sons, Inc., New York, 1986.

[17] Kalnins E.G., Miller W. Jr., Williams G.C., Matrix operator symmetries of the Dirac equation and separation of variables, J. Math. Phys. 27 (1986), 1893-1900.

[18] Kalnins E.G., Miller W. Jr., Williams G.C., Recent advances in the use of separation of variables methods in general relativity, Philos. Trans. Roy. Soc. London Ser. A 340 (1992), no. 1658, 337-352.

[19] Kamran N., McLenaghan R.G., Symmetry operators for neutrino ans Dirac fields on curved spacetime, Phys. Rev. D 30 (1984), 357-362.

[20] McLenaghan R.G., Rastelli G., Separation of variables for systems of first-order partial differential equations and the Dirac equation in two-dimensional manifolds, in Symmetries and Overdetermined Systems of Partial Differential Equations, IMA Vol. Math. Appl., Vol. 144, Editors M. Eastwood and W. Miller Jr., Springer, New York, 2008, 471-496.

[21] McLenaghan R.G., Smith S.N., Walker D.M., Symmetry operators for spin-1/2 relativistic wave equations on curved space-time, R. Soc. Lond. Proc. Ser. A Math. Phys. Eng. Sci. 456 (2000), 2629-2643.

[22] McLenaghan R.G., Spindel Ph., Quantum numbers for Dirac spinor field on a curved space-time, Phys. Rev. D 20 (1979), 409-413.

[23] Miller W. Jr., Symmetry and separation of variables, Encyclopedia of Mathematics and its Applications, Vol. 4, Addison-Wesley Publishing Co., Reading, Mass. - London - Amsterdam, 1977.

[24] Miller W. Jr., Mechanism for variable separation in partial differential equations and their relationship to group theory, in Symmetries and Nonlinear Phenomena (Paipa, 1988), World Scientific, Singapore, 1988, $188-221$.

[25] Smith S.N., Symmetry operators and separation of variables for the Dirac equation on curved space-times, Ph.D. Thesis, University of Waterloo, 2002. 